\title{
El proceso contable según las normas internacionales de información financiera

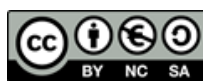

\begin{abstract}
The accounting processes according to international financial information regulations
\end{abstract}

Letty Karina Elizalde. ${ }^{1}$

Recibido: 13-06-2019 / Revisado: 18-07-2019 /Aceptado: 09-08-2019/ Publicado: 06-09-2019

\begin{abstract}
.
DOI: https://doi.org/10.33262/cienciadigital.v3i3.3.813

This article aims to analyze the accounting processes according to international financial reporting standards in business organizations, to meet this purpose a methodology of documentary type is used, with bibliographic design, different sources were consulted, gathering relevant information, highlighting the processes Accountants, elements of the accounting processes, steps of the accounting process, the International Financial Reporting Standards, among others, the reading and interpretation of the texts was carried out in order to formulate an analysis and final reflections of the content. To consolidate the information, a previously elaborated database is used. In the process of the analysis stage of the documents, sources or data, we proceeded to develop the textual content sheets, which allow the handling of the data. Achieving establish that the accounting processes have benefited significantly with the incorporation of International Financial Reporting Standards in business organizations. Likewise, the importance of the International Financial Reporting Standards in the entire financial sphere in an organization becomes evident.
\end{abstract}

Keywords: Accounting process, International Financial Reporting Standards, business organizations.

\footnotetext{
${ }^{1}$ Escuela Superior Politécnica de Chimborazo, Facultad de Administración de Empresas. Universidad de Los Andes de Mérida-Venezuela. Riobamba-Ecuador. letty.elizalde@espoch.edu.ec
} 


\section{Resumen.}

Este artículo tiene como objetivo analizar el proceso contable según las normas internacionales de información financiera en las organizaciones empresariales, para cumplir con dicho propósito se utiliza una metodología de tipo documental, con diseño bibliográfico, se consultaron diferentes fuentes, recopilando información relevante, resaltando los procesos contables, elementos de los procesos contables, pasos del proceso contable, las Normas Internacionales de Información Financiera, entre otros, fue realizada la lectura e interpretación de los textos a los fines de formular un análisis y reflexiones finales del contenido. Para consolidar la información, se utiliza una base de datos previamente elaborada. En el proceso de la etapa de análisis de los documentos, fuentes o datos, se procedió a desarrollar las fichas de contenido textual, que permiten el manejo de los datos. Logrando establecer que los procesos contables se han beneficiado de manera importante con la incorporación de las Normas Internacionales de Información Financiera en las organizaciones empresariales. Así mismo se hace evidente la importancia de las Normas Internacionales de Información Financiera en todo el ámbito financiero en una organización.

Palabras claves: Proceso contable, Normas Internacionales de Información Financiera, organizaciones empresariales.

\section{Introducción.}

La globalización y la integración de los mercados hace necesaria una armonización en la información financiera, que las entidades de los diferentes países proporcionan a sus inversores, analistas y a cualquier tercero interesado, por lo tanto, el objetivo perseguido con la aplicación de un único grupo de normas para todas las entidades que coticen en un mercado, es la obtención de una grado apropiado de comparabilidad de los estados financieros, así como, un aumento de la trasparencia de la información.

La consolidación de mercados internacionales ha obligado a los países a crear normas solidas que les permitan competir económicamente y generar dinámicas cambiarias de alcance mundial. No obstante, para lograr esa tarea, cada una de las naciones ha tenido que evolucionar en su estructura contable.

En este sentido, para Delgado (2009), la estructura contable, visualizada en los estados financieros, son los parámetros que han probado ser los mejores instrumentos de medición de la gestión administrativa para las actuales organizaciones lucrativas, son las calificaciones 
que los decidores obtienen en la complicada selva del mercado, y que mejor sería la uniformidad de los mismos.

Este trabajo está organizado en cuatro secciones distribuidas de la siguiente manera: La primera sección ofrece una introducción al tema donde es expuesta la motivación del estudio. La segunda sección explica la metodología empleada en la porción empírica del estudio; se pone especial énfasis en explicar las decisiones del diseño de la investigación y la medición de las variables. La tercera sección, es una revisión de la literatura que ubica este estudio dentro de la bibliografía de la gestión contable; se ofrece una descripción del modelo general explicitando los contrastes o posturas. La cuarta sección presenta las conclusiones por objetivos, de una manera resumida.

\section{Metodología.}

Esta investigación fue desarrollada de tipo documental, ya que se concentró exclusivamente en la compilación de información en diversas fuentes, examinar documentos escritos referentes a la gestión contable como proceso para maximizar el aprovechamiento de los recursos en las empresas.

En este sentido, de acuerdo a Hernández et al., (2017), la investigación de tipo documental depende fundamentalmente de la información que se obtiene o se consulta en documentos, entendiendo por estos, todo material al que se pueda acudir como fuente de referencia, sin que se altere su naturaleza o sentido, las cuales aportan información o dan testimonio de una realidad o un acontecimiento.

Por otro lado, según Pelekais et al., (2015), para el desarrollo de la investigación documental, se requiere, como condición necesaria, un tema seleccionado, delimitado, justificado, producto de la documentación o de la reflexión personal. Igualmente, es planteado un marco de referencia preliminar que permite orientar la recolección de la información, como la redacción posterior del informe de la investigación. Para el desarrollo, propiamente dicho, es imprescindible ser preciso, claro y sintético, lo cual puede permitir abordar sólo lo contemplado, pertinente, que responda a los propósitos de la investigación. Finalmente, para la redacción se intima agotar varias versiones, experimentar, totalmente, el proceso de escritura.

Asimismo, está enmarcado en un diseño bibliográfico, basándose en la exploración metódica del documental recopilado referente a la gestión contable, con la finalidad de realizar el respectivo análisis.

Igualmente, Sabino (2014), señala que el diseño bibliográfico, se establece cuando los datos a emplear han sido recolectados en otras investigaciones y son conocidos mediante los informes correspondientes a datos secundarios obtenidos por otros, elaborados y procesados de acuerdo a los fines de quienes los manejan. 
Para la consolidación de la información, se utiliza una base de datos elaborada para tal efecto, en la que se guarda lo recabado. En el desarrollo de la etapa de análisis de los documentos, fuentes o datos, se procedió a elaborar las fichas de contenido textual.

En este sentido, el procedimiento de la organización de estos contenidos, así como la revisión esquemática, permite verificar si el proceso de investigación es acertado, además de valorar el material recopilado, así como la detección de excesos en las ideas transcritas, con el fin de organizar o uniformar la investigación de manera eficaz, para constatar que no falten datos esenciales. Tras el proceso anterior, se clasifica el material recopilado siendo depurados aquellos datos con mayor relevancia para responder al objetivo de estudio.

\section{Resultados.}

A los fines de analizar los aspectos contenidos en este artículo, se hace una revisión bibliográfica de los términos que conforman este trabajo:

\section{Proceso contable}

Para Catacora (2012), el proceso contable son todas aquellas consecuencias de pasos e instructivos que se utilizan para el registro de las transacciones u operaciones que realiza una empresa en los libros de contabilidad. De igual manera, se define como la operación diaria ordinaria de un sistema particular de cuentas.

Por otro lado, el proceso contable es reseñado por Revilla (2011), como el manejo de registros que constituyen una fase o procedimientos de la contabilidad. El mantenimiento de los registros conforma un proceso de extrema importancia, toda vez que el desarrollo eficiente de las otras actividades contables depende en alto grado, de la exactitud e integridad de los registros de la contabilidad.

De igual modo, para Espiñeira (2010), los procedimientos contables son una operación diaria de un sistema particular de cuentas, es decir, es un registro de operaciones realizadas en un sistema de contabilidad, los cuales están referidos a cuentas, cargos, créditos a las mismas, activos, pasivos y capital, resumen del funcionamiento de los débitos, créditos, registro de las operaciones, cuentas por cobrar y pagar, el diario, el mayor con sus pases, determinación de las cuentas, balanza de comprobación.

En consecuencia, realizado el análisis teórico se puede inferir que, el proceso contable es el comienzo de una estructura, el cual esta concatenado mediante transacciones o registros diarios que se plasman en los libros de contabilidad de una organización empresarial. Así mismo, se puede argumentar, que los procesos contables, son los pasos, instrucciones o guías, utilizados para el manejo y análisis de la cuentas de los estados financieros.

\subsection{Elementos del proceso contable}


De acuerdo a Catacora (2012), la contabilidad mide los cambios básicos de los estados financieros en tres elementos del proceso como son, contable, activo, pasivo y patrimonio, en este sentido, para medir los cambios que suceden en cada uno de ellos, la contabilidad recurre a la ecuación contable. Los activos de una entidad están constituidos por todos los bienes y derechos a una fecha determinada. Los pasivos están constituidos por todas las deudas y obligaciones de la empresa. El patrimonio representa la participación de los propietarios en los recursos de una entidad.

En este sentido, para Revilla (2011), se presentan a los elementos del proceso contable como un sistema total, donde su desarrollo contará con el apego al conjunto de principios contables de aceptación general, procedimientos preestablecidos para el desarrollo del proceso del registro contable de las actividades, registros y formularios legales, además del plan de cuentas, el cual es detalle de las diferentes cuentas que se utilizan para registrar las transacciones que efectúe la empresa. Por lo tanto, deberán existir un número suficiente de cuentas para reflejar los activos, pasivos, pérdidas y ganancias que se vayan presentando de acuerdo a las actividades desarrolladas por la empresa.

Por otra parte, Espiñeira (2010), describe los bienes, derechos y obligaciones que forman parte del patrimonio de una empresa. Siendo cinco elementos básicos que componen la contabilidad; activo, pasivo, capital o patrimonio, gastos e ingresos. Todos deben seguir un itinerario lógico para su adecuado registro contable, cuyos pasos son: definición, reconocimiento y medición, los cuales quedan reflejados en los estados financieros.

En consecuencia, como resultado del análisis realizado a los planteamientos formulados por los autores estudiados, donde hacen referencia a los elementos del proceso contable, conformados por activos, pasivos y patrimonio de una empresa, los cuales deben pasar por unos pasos de trasformación durante su registro. Se infiere, que los egresos e ingresos también son elementos fundamentales, debiendo ser incorporados a los cambios establecidos, concordando en este sentido con lo expuesto en el texto.

\subsection{Pasos del proceso contable}

Según Bravo (2011), el proceso contable denominado también ciclo contable constituye la serie de pasos o la secuencia que sigue la información contable desde el origen de la transacción hasta la presentación de los Estados Financieros.

Para el autor referido el ciclo contable contiene: Comprobantes o documentos fuente; estado de situación inicial, libro diario, libro mayor, libros auxiliares, balance de comprobación, hoja de trabajo, ajustes y resultados, cierre de libros, estados financieros.

También Catacora (2012), denomina ciclo contable o proceso contable, al conjunto de registros contables efectuados en los diferentes libros de contabilidad con origen en las operaciones que realiza la empresa y que afectan a su patrimonio en un ejercicio económico. Durante el ejercicio económico se deben elaborar distintos documentos que se integrarán en los libros contables. Estos documentos son: 1) Balance de situación, 2) Asiento de apertura, 
3) libros mayores, 4) Asiento de operaciones, 5) Operaciones previas a la determinación al resultado de la empresa, 6) Asientos de regulación, 7) Asiento de cierre, 8) Balance de situación, 9) Cuentas anuales.

A continuación se realiza el respectivo análisis de las distintas fases o etapas que conforman un proceso contable de acuerdo a Catacora (2012). Para ello primero se distinguen los siguientes elementos de análisis:

\subsubsection{Inicio de los registros contables}

Para el autor referenciado, el ejercicio económico de una empresa, normalmente, salvo en empresas puntuales, tiene lugar del 01 de enero al 31 de diciembre. Para ello, las empresas disponen de un patrimonio, que está en el balance de situación de la organización. Este patrimonio viene del ejercicio anterior al que se está abriendo. Es decir, la empresa cuenta al principio del ejercicio con los mismos bienes, derechos y obligaciones que tenía al finalizar el ejercicio anterior. Esta información se tiene en el último asiento del libro diario del ejercicio anterior y en el balance, que representa la situación patrimonial de la empresa al final de ese periodo.

En un nuevo ejercicio económico, se debe reiniciar la contabilidad que se cerró en el ejercicio anterior.

En esta fase del proceso contable se deben realizar los siguientes registros:

- Balance de situación inicial. Que como viene comentando el autor referenciado anteriormente, recoge los bienes, derechos y obligaciones que tiene la empresa al final del ejercicio económico precedente y que coincide con los elementos patrimoniales con los cuales parte la empresa en el nuevo ejercicio económico.

- Asiento de apertura. Con este asiento se procede a la apertura del libro diario. En este asiento, se deben anotar todas aquellas cuentas que aparecen en el balance inicial, teniendo en cuenta que aquellas con saldo deudor van al debe del asiento y las que tienen saldo acreedor van al haber del asiento.

- Apertura de los libros mayores. A la vez que se van realizando los apuntes en el libro diario, se van elaborando los libros mayores de las cuentas.

\subsubsection{Registros correspondientes a las operaciones realizadas}

Prosigue el autor afirmando que el asiento de operaciones, consiste en ir anotando en el libro diario los asientos correspondientes a las operaciones realizadas por la empresa a lo largo del ejercicio. Ordenadas cronológicamente. Estos datos se tienen que anotar también en los mayores correspondientes.

\subsubsection{Cierre de la contabilidad}

Finalmente, el anterior autor referenciado, expresa que las operaciones previas a la determinación del resultado de la empresa, una vez terminado el ejercicio económico, son: 
- Contabilización de las variaciones de existencias

- Periodificación contable

- Contabilización de las provisiones y de las pérdidas por deterioro

- Contabilización de las amortizaciones

- Asientos de regularización. El resultado del ejercicio viene dado por la diferencia entre las cuentas de gastos, grupo 6 y la de ingresos, grupo 7.

A tal efecto, todas las cuentas de gasto se anotarán en el_haber del asiento, para que pasen a tener un saldo nulo, contra la cuenta de resultado del ejercicio. Mientras que las cuentas de ingreso se registrarán en él debe del asiento, contra la cuenta de resultado del ejercicio. Una vez que se haya realizado este asiento, ya se está en disposición de conocer el resultado del ejercicio. Ya que este es el saldo de la cuenta resultado del ejercicio.

- Asiento de cierre. Al analizar los mayores de las cuentas que han ido abriendo a lo largo del ejercicio económico, puede verse cómo sólo las cuentas de activo, pasivo y patrimonio neto tienen saldo. Por lo tanto, para que la contabilidad quede cerrada, todas las cuentas deben tener saldo nulo, procediendo a realizar el asiento de cierre. Para ello deben estar cargadas todas las cuentas acreedoras y abonar las cuentas deudoras. Cuando estas anotaciones se pasen al libro mayor, todas las cuentas estarán con saldo cero.

- Balance de situación final. Para realizar el balance de situación, hay que poner las cuentas que aparecen en el haber del asiento de cierre, en el activo del balance y las que aparecen en él debe, en el pasivo. Este balance de situación final, será el balance de situación inicial del ejercicio siguiente.

En consecuencia y tomando en consideración lo expuesto por los autores arriba analizados, se puede inferir que los pasos del proceso contable están conformados por una serie de etapas o ciclos que deben ser cumplidos al pie de la letra, cada uno posee un propósito diferente, pero al concatenarlos generan información financiera de las entidades económicas. Por lo tanto, es importante para cualquier empresa seguir a cabalidad la información enunciada.

\subsection{Procesos contables en las organizaciones empresariales}

De acuerdo a Jiménez (2015), la información contable en las organizaciones empresariales es una fuente de oportunidades para la gestión administrativa, fiscal, contable, además de financiera; la contabilidad como sistema de información, a partir de los años noventa, ha sido producto no sólo de las exigencias de las organizaciones empresariales en el orbe global, también, de las organizaciones reguladoras que en la urgencia de dar cuenta de estos nuevos requerimientos, ha propiciado la creación de los estándares internacionales de información financiera, que ha permitido normalizar la presentación de estados financieros a nivel mundial.

Complementa lo expuesto, Agreda Palacios (2013), para quien el proceso de globalización de las economías, así como el uso de las tecnologías de la información han introducido 
importantes cambios al entorno financiero, la forma de hacer negocios y el intercambio de información financiera, minimizando barreras derivadas de la distancia física. Cada vez más el dinamismo de los mercados exige mayor y mejor información, por lo tanto, no resulta cuestionable afirmar que, la eficiencia del mercado de capital mundial, depende en gran parte de la disponibilidad y oportunidad de información transparente, comparable y relevante.

De lo expuesto se evidencia que para cualquier organización empresarial, el proceso contable con cada de los elementos que lo conforman, es vital para poder mantener y conocer en tiempo oportuno y real la respectiva situación financiera.

\section{Normas Internacionales de Información Financiera}

Para el Instituto de Estudios Superiores de Administración (IESA 2013), las Normas Internacionales de Información Financiera (NIIF), también conocidas por sus siglas en inglés como International Financial Reporting Standard (IFRS), son normas contables adoptadas por el International Accounting Standards Board (IASB), que establece normas para la presentación de estados financieros entre diferentes países y ha proclamado más de 40 Normas Internacionales de Contabilidad (NIC's).

En este sentido, Para Barrantes (2016), las Normas corresponden a un conjunto único de normas estándar legalmente exigibles y globalmente aceptadas, comprensibles y de alta calidad. Están basadas en principios que permiten que la información incluida en los estados financieros sea comparable y transparente. Esto contribuye a que los inversores y demás grupos de interés que interactúan en mercados de capital de todo el mundo puedan tomar decisiones financieras. Las NIIF son basadas en principios y no en reglas, lo cual requiere en mayor grado la aplicación del juicio profesional por parte de la gerencia en las empresas.

De igual manera, según Vasco (2015), es el conjunto de normas e interpretaciones de carácter técnico, aprobadas, emitidas y publicadas por el Consejo de Normas Internacionales de Contabilidad (Conocido por sus siglas en inglés como el IASB).

En este sentido, se puede inferir, que las NIIF, son unos conjuntos de acuerdos, estatutos, lineamientos, sus cimientos fueron los principios contables, conformadas por organismos reconocidos con una gran experiencia y trayectoria en el área contable a nivel mundial, y poseen la finalidad de unificar criterios a la hora de la presentación de los estados financieros.

\subsection{Objetivos de las Normas Internacionales de Información Financiera}

Los principales objetivos de las NIIF están centrados en generar información de calidad para sus principales usuarios; accionistas, inversionistas, proveedores, clientes y público en general. Otro aspecto no menos importante es la transparencia de la información para el inversor y la comparabilidad de la información financiera. (HRB Consultores, 2018).

En este sentido, Varón (2013), asegura que el objetivo de las NIIF es ayudar a los participantes en los mercados de capitales de todo el mundo y a otros usuarios, a tomar decisiones económicas. Ese es el entorno en el cual giran las NIIF, y en el paradigma de la 
utilidad de la información financiera para la toma de decisiones económicas es en el cual debe ubicarse el elaborador de información financiera.

Por su parte, Rodríguez (2015), considera que el objetivo de esta norma es establecer los procedimientos que se deben seguir cuando una empresa adopta las NIIF por primera vez como base de presentación para sus estados financieros con carácter general.

En consecuencia, y tomando en consideración lo aportado por los autores referenciados, se puede inferir que dentro de los objetivos de las NIIF, hay uno que pudiese ser el principal o general, y es precisamente la uniformidad de criterios o de pasos a seguir para la ubicación, el registro, la elaboración y la presentación en los estados financieros, donde se evidencie la situación económica de las organizaciones empresariales, de esta manera puede ser comprendida en cualquier país del mundo, donde sea requerida.

\subsection{Principales organismos internacionales y países que promueven la adopción de las NIIF}

De acuerdo a HRB Consultores (2018, los principales organismos internaciones y países que promueven la adopción de las NIIF son: Fondo Monetario Internacional, FMI Organización de las Naciones Unidas ONU, Organización Mundial de Comercio, OMC Banco Mundial, BM Banco Interamericano de Desarrollo, BID Organización Internacional de Bolsas de Valores, IOSCO. Por lo tanto, las NIIF son usadas en muchas partes del mundo, entre los que se incluye la Unión Europea, Hong Kong, Australia, Chile, Colombia, Malasia, Pakistán0020, India, Panamá, Guatemala, Perú, Rusia, Sudáfrica, Singapur, Turquía, Ecuador, Venezuela, Nicaragua y El Salvador. Desde el 28 de marzo de 2008, alrededor de 75 países obligan el uso de las NIIF, o parte de ellas. Otros muchos países han decidido adoptar las normas en el futuro, bien mediante su aplicación directa o mediante su adaptación a las legislaciones nacionales de los distintos países.

Igualmente, Varón (2013), reseña que desde el 2002 se ha producido también un acercamiento entre el IASB International Accounting Standards Board y el FASB Financial Accounting Standards Board, entidad encargada de la elaboración de las normas contables en Estados Unidos, para tratar de armonizar las normas internacionales con las norteamericanas. En Estados Unidos las entidades cotizadas en bolsa tendrán la posibilidad de elegir si presentan sus estados financieros bajo US GAAP (el estándar nacional) o bajo NIIF. En consecuencia, las NIIF han sido adoptadas oficialmente por la Unión Europea como sus normas contables, pero sólo después de pasar por la revisión del EFRAG, por lo que para comprobar cuáles son aplicables es necesario comprobar su estatuto oficial.

En consecuencia y tomando como referencia lo planteado anteriormente por los teóricos abordados, las NIIF se han constituido como una norma de aceptación mundial, referidas por la gran mayoría de los países, sirviendo de unificación de los criterios, logrando un entendimiento de las operaciones y transacciones financieras, con la intención de manejar un solo idioma financiero y contable. 


\subsection{Beneficios en la aplicación de las NIIF}

La adopción de las NIIF/IFRS ofrece una oportunidad para mejorar la función financiera a través de una mayor consistencia en las políticas contables, obteniendo beneficios potenciales de mayor transparencia, incremento en la comparabilidad y mejora en la eficiencia. Entre otros pueden citarse: Acceso a mercados de capital, Transparencia en las cifras de los Estados Financieros, Información consistente y comparable, Mismo lenguaje contable y financiero, Reducción de costos, Herramienta para la alta gerencia en la toma de decisiones, Modernización de la Información financiera y Simplificar la preparación de los Estados Financieros (DELOITTE, 2018).

En este sentido, Rubiños (2015), establece que, dentro de los beneficios para la aplicación de las NIIF, están:

$\mathrm{Su}$ aceptación es mundial, evitando costos y tiempo adicional en procesos de conversión y preparaciones de nuevos estados financieros. 2) Es un lenguaje financiero uniforme aceptado en el mercado de capitales. 3) Las NIIF son constantemente actualizadas, evaluadas y adaptadas conforme a la evolución de los negocios. 4) Facilita acceder al mercado internacional, para atraer potenciales clientes o proveedores o financistas o inversionistas. 5) Facilita el "benchmarking" financiero con entidades del mismo sector de diferentes países. 6) Las amplias revelaciones dan confianza a los inversionistas y otros usuarios externos. 7) Facilita la toma de decisiones. 8) Requiere presentar información comparativa y consistente de año en año. 9) No dependen de lo legal o tributario, sino de la realidad económica de la entidad. 10) Facilita la supervisión y el control de los reguladores. 11) Reduce el tiempo para administrar temas financieros. 12) Facilita la consolidación entre grupos económicos, incluso si son de varios países. 13) No se limitan a un marco legal de una jurisdicción. 14) Son normas de fácil aplicación en la práctica comparadas con otros marcos contables 15) Disminuyen la percepción del riesgo (Espiñeira, 2010).

Tomando en consideración lo expuesto por los autores arriba señalados, se puede inferir, que la aplicación de las NIIF genera amplios beneficios, viene a organizar el proceso contable, con la finalidad que exista un solo criterio o lenguaje para ayudar a la uniformidad de los mismos, globalizando todos los problemas que pudiesen presentar a la hora de los registros contables, mostrando unos resultados que pudiesen ser comprendidos por toda la comunidad empresarial a nivel mundial.

\subsection{Impactos de la implementación de las NIIF en el proceso contable}

Según Salazar (2017), a nivel contable el impacto se evidencia en el plan de cuentas - PUC, las operaciones, los registros, los comprobantes y la modificación de los estados financieros. La actualización para los profesionales de la contaduría pública se convierte en una necesidad, porque las Normas Internacionales de Información Financiera - NIIF hoy son 
tendencia. Por tal razón la mayoría de las universidades ofrecen cursos de implementación de las normas o simplemente fue incluida como una materia más en los pensum académicos. En síntesis, existen condiciones técnicas para entender y aplicar los estándares internacionales.

En este sentido, Vásquez (2013), asegura que existen las siguientes falencias de conocimiento profesional: a) La diferencia entre costo, precio y valor. b) El sentido del mantenimiento de capital como base de generación de ganancias. c) La diferencia entre un recurso, un bien y un derecho. d) La diferencia entre valores de cambio y valores de uso y sus metodologías de determinación. e) El concepto de obligación implícita. f) El criterio de reconocimiento. g) El sentido de beneficio económico futuro y deterioro de capacidad de generación. h) El concepto de unidad generadora de efectivo y segmento de operación. i) Las bases de medición y el concepto de valor razonable. j) El riesgo y el beneficio implícito de un hecho económico. k) La relación de flujo de efectivo con la base de causación. 1) La diferencia entre vida útil y vida económica de un recurso.

Para Restrepo y Muñoz (2011), algunas de sus funciones están en "conocer, estudiar, discernir y determinar su contenido, impacto y relación con el modelo colombiano, sus procesos y procedimientos para la manipulación de la información" (p.36). Por lo tanto la profesión contable exige cada día estar actualizado y capacitado en lo que a su campo de acción se refiere.

\section{Probabilidad de beneficios futuros}

De acuerdo a las Normas Internacionales de Información Financiera (NIIF 2009), la probabilidad de beneficios futuros, es el criterio importante en el reconocimiento de una partida en los estados financieros, la evaluación del grado de incertidumbre o riesgo, se elabora para partidas individuales significativas, que pueden ser una unidad de negocios, un segmento, una sucursal, entre otros.

De igual manera, para Espiñeira (2010), se expone que la probabilidad se utiliza, en las condiciones para su reconocimiento, con referencia al grado de incertidumbre con el cual los beneficios económicos futuros asociados al mismo, llegan o saldrán de la entidad, este concepto tiene en cuenta la incertidumbre que caracteriza el entorno en que opera la empresa, la medición del grado de incertidumbre, correspondiente al flujo de los beneficios futuros, se hace a partir de la evidencia disponible cuando se preparan los estados financieros.

Por su parte, la International Financial Reporting Standard (2009), expone la probabilidad que cualquier beneficio económico futuro asociado con el elemento, fluya desde la entidad. En este sentido, la valoración del grado de incertidumbre que se asigna al flujo de los beneficios económicos futuros se hace con base a las evidencias relacionadas con las condiciones al final del periodo.

En consecuencia y tomando como premisa lo expuesto por los autores arriba mencionados, se puede inferir, que la probabilidad de beneficios futuros, es uno de los principios más 
relevantes, dado que es aquel grado de incertidumbre enmarcado hacia los futuros beneficios. Este procedimiento es valioso de elaborar y requiere de una asesoría financiera, de tal manera que es un esfuerzo mayor para una organización empresarial y se debe optar por otro criterio de evaluación.

\section{Confiabilidad en la medición}

Catacora (2012), indica que cuando el costo o valor debe estimarse, el uso de estimaciones razonables es una parte esencial de la preparación de los estados financieros y no debe menoscabar su fiabilidad. No obstante, cuando no puede hacerse una estimación razonable, la partida no se reconoce en el balance ni en el estado de resultados.

Igualmente, Espiñeira (2010), la partida, tiene un costo o valor que puede ser medido de manera confiable, la segunda condición para el reconocimiento de las partidas es que posean un costo o valor que pueda ser medido de forma fiable. En muchos casos, cuando el costo o valor deba estimarse, el uso de estimaciones razonables es una parte esencial de la preparación de los estados financieros, y no deben menoscabar su fiabilidad. En relación, cuando no sea posible una medición sensata, la cuenta no será registrada en los balances.

En función de lo planteado, de acuerdo a las Normas Internacionales de Información Financiera (NIIF 2009), el elemento contiene un costo o valor que se puede medir confiablemente, en muchos casos estas partes son conocidas o en otros casos tienen que estimarse. Por ello, el uso de estimaciones razonables es parte esencial de la preparación de los estados financieros y no deteriora su confianza, cuando no es posible establecer una estimación, la partida no será reconocida en el resultado.

En consecuencia y tomando en consideración la expuesto por los autores arriba mencionados, se puede inferir, que la confiabilidad en la medición de forma razonable, es aquella que los resultados de dicha medición sean sistemáticamente precisos, esto requiere que el instrumento usado para realizar la medición de aquellos elementos que no son reconocidos en los estados financieros, siendo así la estimación parte fundamental para la preparación de los mismos.

\section{Medición de los elementos de los estados financieros}

Para Catacora (2012), la medición es el procedo de determinación de los importes monetarios por lo que se reconocen que llevan contablemente los elementos de los estados financieros, para su inclusión en el balance y estado de resultado, es necesario la selección de una base o método particular de medición. En los estados financieros se emplean diferentes bases de medición, con diferentes grados en distintas combinaciones entre ellas, tales bases o métodos son: costo histórico, costo corriente, valor realizable y valor presente.

En este sentido, según las Normas Internacionales de Información Financiera (NIIF 2009), se exponen técnicamente y se refiere a la medición de activos, pasivos, ingresos y gastos. Por 
lo tanto, medición es el proceso de determinación de las cantidades monetarias a las cuales la entidad mide, en sus estados financieros, los activos, pasivos, ingresos y gastos.

Igualmente, para Estupiñán (2012), en los estados financieros se emplean diferentes bases de medición, con diferentes grados y en distintas combinaciones entre ellas. El método de medición, continua el autor, comúnmente utilizado por las entidades, es el costo histórico este se combina con otras bases de medición, por ejemplo, los inventarios se llevan contablemente al menor valor entre el costo histórico y el valor neto realizable.

Partiendo de los supuestos anteriores, se puede inferir, que la medición viene siendo un proceso efectuado para determinar los importes monetarios por los cuales serán reconocidos los elementos de los estados financieros, en otras palabras, establecer un valor a las partidas de los estados financieros aplicando las bases de medición permitidas por las NIIF, esta medición por un costo histórico, costo corriente, valor realizable, y un valor actual.

\section{Criterios a implementar para la aplicación de las NIIF}

En este sentido, de acuerdo al autor anteriormente referenciado, existen algunos criterios de reconocimiento y valoración que se deben utilizar y considerar al momento de la implementación de las NIIF, los cuales se describen a continuación: aceptar todos los activos y pasivos cuyo reconocimiento es requerido, no aceptar en el balance inicial partidas de activos o de pasivos en los cuales el reconocimiento no es permitido, reclasificar partidas que eran reconocidas bajo requerimiento locales como de algún tipo de activo, pasivo o componente del patrimonio. Aplicar todas las NIIF al medir todas las partidas en el balance inicial.

Por su parte, según las Normas Internacionales de Información Financiera (NIIF 2009), la entidad las aplicará, si sus primeros estados financieros con arreglo a las NIIF corresponden a un ejercicio que comience a partir del 1 de julio del 2009, por lo tanto, se permite su aplicación anticipada, la entidad emplea sus modificaciones en los ejercicios anuales que comiencen en la fecha arriba descrita. Si una entidad aplicase la NIC 23 Costos por préstamos en ejercicios anteriores, esas modificaciones se aplicarían también a esos ejercicios.

De igual manera, Catacora (2012), expone los criterios que se deben de implementar en la adopción de las NIIF: aceptar todas las partidas contables cuyo reconocimiento es requerido, no aceptar en el balance inicial operaciones cuyo reconocimiento no sea permitido, reclasificar operaciones contables bajo reconocimiento locales y aplicar las NIIF, al medir todas las cuentas en el balance inicial, así mismo, reconocer las políticas contables que deben ser establecidas al momento de la implementación de dicha norma.

Con lo expuesto por los autores analizados con anterioridad en el texto, se infiere que dentro de los criterios que deben ser tomados en consideración al momento de implementar las NIIF, se debe revisar, analizar y preparar el balance de apertura a la fecha de transición en el cual se hará el reconocimiento de los activos, pasivos y patrimonio de la entidad, así como 
también, se requiere tomar en consideración las políticas contables plasmadas en las NIIF, las cuales emanan del proceso que se debe aplicar para la adopción de la misma.

\section{Identificación de las políticas contables aplicables bajo las NIIF}

Según las Normas Internacionales de Información Financiera (NIIF 2009), las organizaciones empresariales deben asegurar que los primeros estados financieros con arreglo a las NIIF de una empresa, así como sus informes financieros intermedios, relativos a una parte del ejercicio cubierto por tales estados financieros, deben contener información de alta calidad que sea trasparente para los usuarios y comparable para todos los ejercicios que se presenten, además suministrar un punto de partida adecuado para la contabilización según las propias NIIF y puede ser obtenida a un costo que no exceda a los beneficios proporcionados a los usuarios.

Espiñeira (2010), por su parte, afirma que las empresas deben utilizar las mismas políticas contables en sus balances de apertura con arreglo a las NIIF, y a lo largo de todos los ejercicios que se presenten en sus primeros estados financieros de acuerdo a las NIIF. Estas políticas contables deben cumplir con cada NIIF vigente en la fecha de presentación de sus estados financieros. Para cumplir con la NIC-1 Presentación de estados financieros, los primeros con arreglo a las NIIF de la empresa incluirán, al menos, un año de información comparativa de acuerdo con las Normas Internacionales de Información Financiera.

De igual manera, Catacora (2012), asegura que las empresas deben aplicar durante la transición, los Principios de Contabilidad generalmente aceptados anteriores a las NIIF, asimismo, las entidades usaran las mismas políticas contables en su balance de apertura con arreglo a las NIIF y a lo largo de todos los ejercicios que se presenten en sus primeros estados financieros con arreglos a las NIIF.

En consecuencia y tomando en cuenta los postulados arriba mencionados, se puede inferir, que la identificación de políticas contables tienen la finalidad de contar con las bases necesarias para llevar a cabo el proceso de consolidación de estados financieros de las organizaciones empresariales, por lo tanto, es necesario definir y aprobar políticas contables uniformes tomando en cuenta las NIIF, las cuales presentan los lineamientos a presentar al momento de llevar a cabo los procesos contables.

\section{Preparación del balance de apertura a la fecha de transición NIIF}

Po su parte, se establece que el balance de apertura basado en las Normas Internacionales de Información Financiera (2009), es el punto de partida para todos los subsecuentes periodos contables. A tal efecto, las entidades deben preparar un balance de apertura a la fecha de transición, la cual se refiere, al comienzo del periodo más antiguo para el que la entidad presenta información financiera comparativa completa según las NIIF, para sus primeros estados financieros presentados de conformidad con las normas establecidas. 
Asimismo, para Rodríguez (2010), se establece que todas las entidades que adopten por primera vez las NIIF, deben preparar un balance de apertura con arreglo a las normas internacionales en la fecha de transición. Es decir este es el punto de partida para la contabilización según lo acordado internacionalmente. Por otra parte señala, que dichos estados financieros, no necesitan ser presentados, sin embargo, el mismo es de suma importancia para efectos comparativos, por su relevancia para la adecuada comprensión de los estados financieros del periodo corriente.

De igual manera, Espiñeira (2010), expresa que es el estado, en el que por primera vez, se medirán de acuerdo con el nuevo marco normativo los activos, pasivos y patrimonio de las entidades que apliquen este decreto, su fecha de corte es la fecha de transición. El estado de situación financiera de apertura no será puesto en reconocimiento del público ni tendrá efectos legales en dicho momento.

En este sentido, se puede inferir, que el balance de apertura para la aplicación de las NIIF, es aquel estado donde se presentan los ajustes o principios aplicados según lo establecido en dicha norma. Debiendo constatar que dicho balance no debe ser incluido en los estados financieros de la empresa, debido a que es un balance utilizado para efectos de adopción de las Normas Internacionales de Información Financiera.

\subsection{Considerar y aplicar las exenciones para la aplicación}

Para Estupiñan (2012), una entidad que adopte por primera vez las NIIF puede optar por no aplicar de forma retroactiva las NIIF 3, modificada en el 2008, a las combinaciones de negocios realizadas en el pasado, por lo tanto, a las combinaciones de negocios anteriores a la fecha de transición de las NIIF. Sin embargo, si la entidad que adopte por primera vez las NIIF, re-expresase cualquier combinación de negocios para cumplir con las NIIF 3 modificada en el 2008, re-expresará todas las combinaciones de negocios posteriores y aplicará también la NIC 27 modificada en el 2008, desde esa misma fecha.

En este sentido, se expone que una entidad que adopte por primera vez las NIIF, no aplica las NIIF 3 de forma retroactiva a una combinación de negocios anterior, este hecho tendrá las siguientes consecuencias para esa combinación:

La entidad que adopte por primera vez las NIIF, conservara la misma clasificación como una adquisición por parte de la adquiriente legal, como una adquisición inversa por parte de la entidad legalmente adquirida o como una unificación de interés que tenía en sus estados financieros elaborados con arreglos a los PCGA anteriores. La entidad que adopte por primera vez las NIIF, reconocerá todos sus activos y pasivos en la fecha de transición a las NIIF, que hubieran sido adquiridos o asumidos en una combinación de negocios pasada. (Normas Internacionales de Información Financiera, NIIF 2009).

Por su parte, Catacora (2012), complementa lo anterior y afirma que la entidad que adopte por primera vez las NIIF, excluirá de su estado de situación financiera de apertura con arreglo a las NIIF todas las partidas reconocidas por la PCGA, anteriores, que no cumplan las 
condiciones para su reconocimiento como activos y pasivos según las NIIF, la entidad que adopte por primera vez las NIIF contabilizará los cambios resultantes.

\subsection{Identificar las divulgaciones que requiere la NIIF}

En este sentido, para las Normas Internacionales de Información Financiera (NIIF 2009), la entidad aplicará estas normas, si sus primeros estados financieros con arreglo a las NIIF corresponden a un ejercicio que hubiese iniciado a partir del 1 de julio del 2009, se permite su aplicación anticipada, la entidad debe utilizar las modificaciones de los párrafos D1 (n) y D23 en los ejercicios anuales que hubiese aplicado el 1 de julio del 2009. Si una entidad aplicase la NIC 23 Costos por préstamos revisada 2007 en ejercicios anteriores, esas modificaciones se instrumentan también a esos ejercicios.

Asimismo, para Espiñeira (2010), las divulgaciones se concentran principalmente en los segmentos en el formato primario, presentando solo información limitada en el segmento secundario, las divulgaciones en el formato primario incluyen: ingresos, resultados, activos, pasivos, inversiones en no monetarios, depreciación y amortización, el monto total de los gastos significativos no realizados en efectivo y las pérdidas por deterioro. Las divulgaciones para el segmento secundario incluyen ingresos para los activos y gasto de capital. Por lo tanto, no se requiere mostrar el resultado neto para los secundarios, debe conciliarse la información divulgada por dimensiones con los totales mostrados en los estados financieros.

En acuerdo con los autores antes referidos, se puede inferir, que las divulgaciones para los segmentos secundarios incluyen ingresos por segmentos, activos y gasto de capital. En este sentido, no se requiere mostrar el resultado neto para los segmentos secundarios. Debe conciliarse la información divulgada por segmentos con los totales mostrados en los estados financieros.

\section{Conclusiones}

- Sobre la base de las ideas expuestas, se puede concluir, que el proceso contable es el comienzo de una estructura, el cual esta concatenado mediante transacciones o registros diarios que se plasman en los libros de contabilidad de una organización empresarial, referente a los elementos que lo constituyen, activo, pasivo y patrimonio. Concerniente a las Normas Internacionales de Información Financiera (NIIF), se puede decir que corresponden a un conjunto único de normas estándar legalmente exigibles y globalmente aceptadas, sobre sus objetivos está la uniformidad de criterios o de pasos a seguir para la ubicación, el registro, la elaboración y la presentación en los estados financieros.

- De igual manera, dentro de los principales organismos internacionales y países que promueven la adopción de las NIIF, se encuentran un gran número de ellos que las han aceptado y puesto en práctica, por ello, dentro de los beneficios ofrece una oportunidad para mejorar la función financiera a través de una mayor consistencia en las políticas contables. Como su mayor impacto la exigencia de la actualización como 
profesionales contables o la probabilidad de beneficios futuros es el criterio importante en el reconocimiento de una partida en los estados financieros, por otro lado, la confiabilidad en la medición el mismo, indica cuando el costo o valor debe estimarse.

- Así mismo, la medición de los elementos de los estados financieros es un proceso efectuado para determinar los importes monetarios por los cuales van hacer reconocidos los elementos de los estados financieros, como criterio se debe de tomar en consideración las políticas contables plasmadas en las NIIF, identificando las políticas contables aplicables a las NIIF, preparando el balance a la fecha de transición, considerando y aplicando exenciones para la aplicación, identificando las divulgaciones de las NIIF.

\section{Referencias Bibliográficas.}

Agreda, X. (2013). Normas internacionales de información: Avances en su aplicación en Estados Unidos de América, México y Venezuela. Revista del Centro de Investigación. Universidad La Salle, 10 (39), 181-193.

Barrantes, F. (2016). ¿Qué son las Normas Internacionales de Información Financiera (NIIF)? y las 4 fases para el proceso de convergencia. Documento en línea en: https://blogs.upc.edu.pe/sinergia-e-innovacion/conceptos/que-son-las-normasinternacionales-de-informacion-financiera-niif-y Consultada 07/10/2018.

Bravo, M. (2012). Contabilidad General. Ecuador: Editorial Escobar.

Catacora, F. (2012). Contabilidad. Venezuela: Red Contable Publicaciones.

Delgado, C. (2009). Análisis de los estados financieros. México: Editorial Trillas.

DELOITTE (2018). ¿Qué son las NIIF | IFRS? Documento en línea en: https://www2.deloitte.com/co/es/pages/ifrs_niif/normas-internacionales-de-lainformacion-financiera-niif---ifrs-.html Consultada 07/10/2018.

Espiñeira, S. (2010). Adopción de las VEN-NIIF para PYMES. Venezuela: Pricewaterhouse Coopers.

Estupiñán, R. (2012). Estados Financieros Básicos Bajo NIC/NIIF. Colombia: Eco. Ediciones.

Hernández, R., Fernández C., Baptista P. (2017). Fundamentos de la investigación. México: Editorial Mac Graw-Hill/Interamericana.

Horngren, W. (2007). Comprender las Normas Internacionales de Información Financiera. Barcelona España: Edición Gestión 2000. 
HRB Consultores (2018). Normas Internacionales de Contabilidad Financiera (NIIF). Documento en línea en: https://hbrconsultores.com/art\%C3\%ADculos/f/normasinternacionales-de-contabilidad-financiera-niif Consultada 07/10/2018.

Instituto de Estudios Superiores de Administración (IESA 2013). Normas Internacionales de Información Financiera. Documento en línea en: http://www.iesa.edu.ve/inicio/2013junio-21/1350=normas-internacionales-de-informacion-financiera. ${ }_{-}$Consultado $07 / 10 / 2018$.

International Financial Reporting Standard (2009). Model financial statements. Documento en línea en: https://www.iasplus.com/en/binary/fs/2009modelfs.pdf Consultada 08/10/2018.

Jiménez, R. (2015). Estándares Internacionales de Información Financiera: Algunos efectos en las organizaciones empresariales. Lúmina, [S.1.], n. 16, p. 112-135, dic. 2015. ISSN 2619-6174. Disponible en: $<$ http://revistasum.umanizales.edu.co/ojs/index.php/Lumina/article/view/1671/2904 >. Fecha de acceso: 09 oct. 2018.

Normas Internacionales de Información Financiera (NIIF 2009). Para pequeñas y medianas entidades. NIIF para PYMES. Consejo de Normas Internacionales de Contabilidad. Documento en línea en: https://www.mef.gob.pe/contenidos/conta_publ/con_nor_co/vigentes/niif/NIIF_PY MES.pdf Consultada 07/10/2018.

Normas Internacionales de Información Financiera 8 (NIIF 8, 2017). Comité permanente de principios de contabilidad de la Federación de Colegios de Contadores Públicos de Venezuela. Criterios para la aplicación en Venezuela de los VEN-NIF PYME, (BA VEN NIF-8). Venezuela. Documento en línea en: http://www.fccpv.org/files/vennif/20170330_BA_VEN-NIF_Nro_8_V-4.pdf Consultada 07/10/2018.

Pelekais, C; El Kadi, O; Seijo, C; Neuman, N (2015). El ABC de la Investigación. Guía Didáctica. Maracaibo. Venezuela: Ediciones Astro Data S.A.

Restrepo, J., \& Muñoz L. (2011). Colombia: Hacia la adopción y aplicación de las NIIF y su importancia. (Trabajo de grado, Universidad de Antioquia,). Recuperado de: https://aprendeenlinea.udea.edu.co/revistas/index.php/adversia/article/viewFile/109 54/10047 Consultado 07/10/2018.

Revilla, L. (2011). Adopción de VEN/NIF PYME. Venezuela: Baker Tilly.

Rodríguez, A. (2010). Adopción por primera vez de las NIIF. Un análisis teórico de la norma. Actualidad Contable Faces. Venezuela. 
Rodríguez, P. (2015). Objetivos de las NIC/NIIF. Documento en línea en: http://patriciarodriguez.blogspot.com/2005/08/objetivos-de-las-nicniif.html Consultada 07/10/2018.

Rubiños, W. (2015). Aportes y Ventajas de las Normas Internacionales de Información Financiera - NIIF. Documento en línea en: http://ucsp.edu.pe/wpcontent/uploads/2015/11/Wilfredo-Rubi\%C3\%B1os-Aportes-y-Ventajas-de-lasNIIF.pdf Consultada 07/10/2018.

Sabino, C., (2014). El proceso de investigación. Caracas Venezuela: Editorial Panapo.

Salazar, L. (2017). Impacto de la implementación de las normas internacionales de información financiera en la profesión contable. Documento en línea en: https://repository.unimilitar.edu.co/bitstream/10654/17066/1/SalazarGomezLadyYe raldin2017.pdf Consultada 07/10/2018.

Tamayo, A. y Escobar P. (2008). Análisis de estados financieros. Fundamentos y aplicación. Barcelona España: Ediciones Gestión 2000.

Torre, M. (2010). Resumen de las VEN-NIF para PYMES. KPMG. Venezuela.

Varón, L. (2013). Que comprenden las NIIF. Documento en línea en: https://www.gerencie.com/que-comprenden-las-niif.html Consultada 07/10/2018.

Vasco, F. (2015). Normas Internacionales de Información Financieras. Documento en línea en:

http://www.camaramedellin.com.co/site/Portals/0/Documentos/Biblioteca/memorias /normas-internacionales-de-financiera.pdf Consultada 07/10/2018.

Vásquez, R. (2013). Revista internacional Legis de contabilidad y auditoría. Tras la verdad de los impactos de las NIIF en el sector empresarial. Recuperado de: http://www.comunidadcontable.com/BancoConocimiento/N/noti- Consultada 07/10/2018. 


\section{PARA CITAR EL ARTÍCULO INDEXADO.}

Elizalde, L. (2019). El proceso contable según las normas internacionales de información financiera. Ciencia Digital, 3(3.3), 254-273. https://doi.org/10.33262/cienciadigital.v3i3.3.813

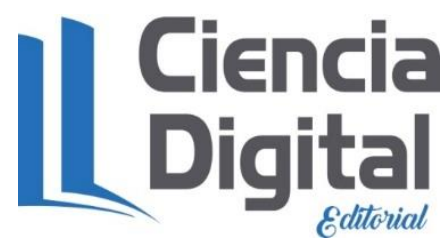

El artículo que se publica es de exclusiva responsabilidad de los autores y no necesariamente reflejan el pensamiento de la Revista Ciencia Digital.

El artículo queda en propiedad de la revista y, por tanto, su publicación parcial y/o total en otro medio tiene que ser autorizado por el director de la Revista Ciencia Digital.
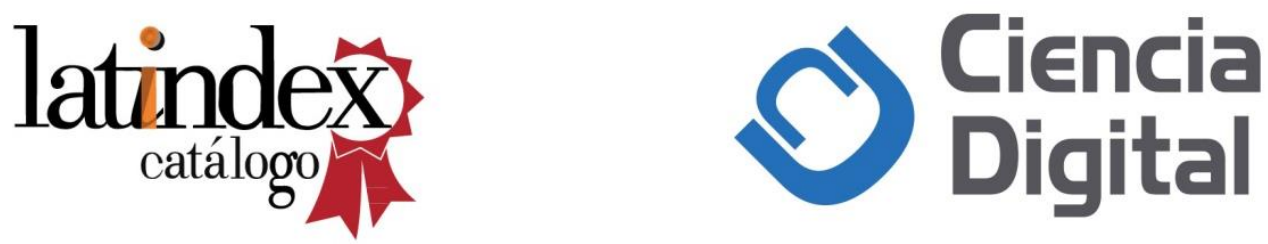